\title{
Leveraging the Near-Far Effect for Improved Spatial-Reuse Scheduling in Underwater Acoustic Networks
}

\author{
Roee Diamant, Member, IEEE, Paolo Casari, Senior Member, IEEE, \\ Filippo Campagnaro, Student Member, IEEE, Michele Zorzi, Fellow, IEEE
}

\begin{abstract}
We present a spatial reuse resource allocation scheme for underwater acoustic networks that organizes communications so as to avoid destructive collisions. One prime source of collisions in underwater acoustic networks is the so called near-far effect, where a node located farther from the receiver is jammed by a closer node. While common practice considers such situation as a challenge, in this paper we consider it as a resource, and use it to increase the network throughput of spatial reuse time-division multiple access. Our algorithm serves two types of communications: 1) contention-free and 2) opportunistic. Our objective is to miximize the time slot allocation while guaranteeing a minimum per-node packet transmission rate. The result is an increase in the number of contentionfree packets received, and a decrease in the scheduling delay of opportunistic packets. Numerical results show that, at a slight cost in terms of fairness, our scheduling solutions achieve higher throughput and lower transmission delay than benchmark spatial-reuse scheduling protocols. These results are verified in a field experiment conducted in the Garda Lake, Italy, where we demonstrated our solution using off-the-shelf acoustic modems. To allow the reproducibility of our results, we publish the implementation of our proposed algorithm.
\end{abstract}

Index Terms-Underwater acoustic networks, near-far effect, spatial-reuse scheduling, time-division-multiple-access (TDMA), long propagation delay, optimization, simulation, sea trial, DESERT Underwater

\section{INTRODUCTION}

The design of medium access control (MAC) protocols for underwater acoustic networks (UWANs) faces several challenges [1], [2], usually summarized into the concept of space-time uncertainty. The significant delays induced by the low propagation speed of underwater acoustic signals imply that channel access decisions are not optimal when informed only by instantaneous channel sensing. Rather, a considerable amount of wait time would be required to safely gain channel access and ensure collision avoidance [3]. Otherwise, packet collisions are possible, where a collision is defined as the superposition of one or more packets at the receiver, possibly impeding the correct reception of some or all of them.

We consider time-division multiple access (TDMA)-based scheduling, which largely fits UWANs with high demand for packet transmission rates [4]. Our system model includes

Manuscript received: January 20, 2016; revised May 23 and October 15, 2016; accepted December 19, 2016. The associate editor coordinating the review of this paper and approving it for publication was A. Zajic.

R. Diamant (corresponding author, email: roeed@univ.haifa.ac.il) is with the Department of Marine Technology, University of Haifa, Israel. Paolo Casari is with the IMDEA Networks Institute, Madrid, Spain. F. Campagnaro and M. Zorzi are with the Department of Information Engineering, University of Padova, Italy.

This work has been supported in part by the US Office of Naval Research under Grant no. N62909-14-1-N127. Part of this work has been presented at MTS/IEEE OCEANS, Shanghai, China, Apr. 2016.

Digital Object Identifier: XX.XXXX/XXXXX network nodes transmitting information to a sink, as well as exchanging information among themselves. Considering that UWANs serve for status exchange of information as well as for transmitting occasional data, we consider two types of traffic demands: status packets, whose target is the sink, and whose minimum reception rate must be guaranteed; and opportunistic packets, whose destination can be any node and that are randomly generated and handled on a best-effort basis. This general framework can be embodied by a number of applications, e.g., a team of divers and/or autonomous underwater vehicles (AUVs) performing underwater work. This can be the case for rescue operations for a damaged ship, oil recovery from a wrecked tanker, or industrial work to install or maintain pipes, infrastructures, and communication lines. In all cases, the divers send periodic (P) status reports to a surface vessel, which serves as a sink; in addition, they exchange opportunistic (O) control packets to coordinate and assist one another. The former are served in a contentionfree fashion, whereas the latter are transmitted less frequently and at random times, and are served in an opportunistic way. Fig. 1 shows a diagram of this scenario, where some divers are connected to a "cluster head" $(\mathrm{CH})$ diver: the two divers on the left are sending $\mathrm{P}$ messages to the $\mathrm{CH}$; at the same time, another diver on the right transmits an $\mathrm{O}$ message to its peer further down.

In TDMA, to guarantee collision-free reception, each time slot includes a guard interval whose duration is given by the maximum propagation delay in the network plus an additional guard period to compensate for possible clock drifts. Since, in underwater acoustics, propagation delays are much

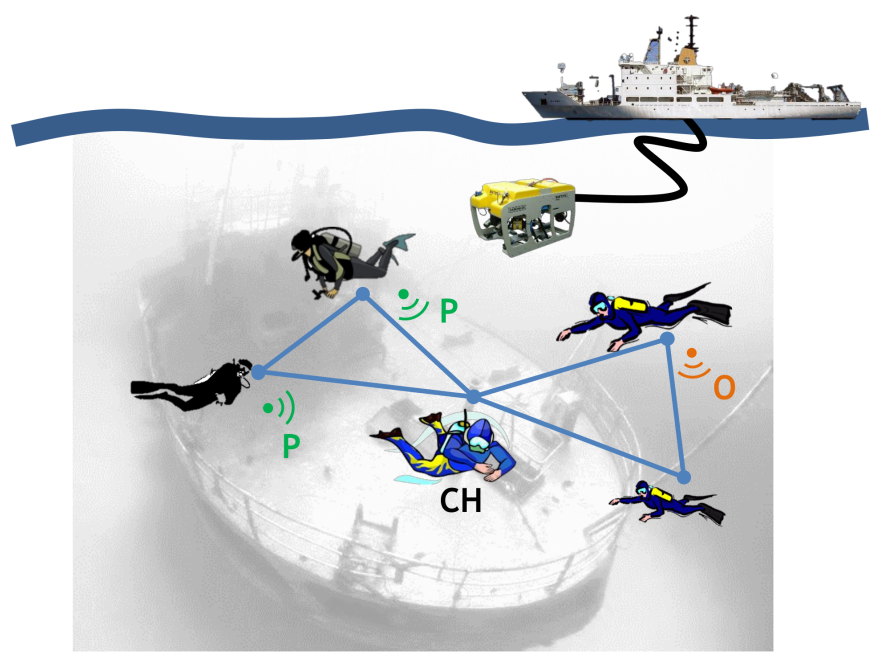

Fig. 1. Sketch of our considered application scenario. 
greater than clock drifts, the network performance is weakly affected by poor time synchronization among the nodes. Still, in TDMA the channel utilization is low. To overcome this problem, a possible solution is offered by spatial-reuse TDMA channel access schemes. Under some assumptions, such as the knowledge of the propagation delays among the nodes [5], [6] or of the local network topology [7], these schemes substantially improve the channel utilization by allowing mutually harmless communication sessions to co-exist. This is achieved either by leveraging on the propagation delays to decouple reception over time at the receiver, or by exploiting different attenuation figures over different distances to ensure that no harmful collisions take place at active receivers.

One of the most common sources of collisions is the so called near-far effect, whereby a node located farther from the receiver is jammed by a closer node. This scenario is very common in UWANs, where the large attenuation per unit distance traveled by an acoustic signal is likely to create nearfar scenarios. In the available literature on MAC protocols for UWANs, the main approach is to avoid near-far events by designing protocols so that the collision of a signal by a "near" node on the transmission by a "far" node can be avoided [6], or at least limited to a minimum. The latter has been achieved, e.g., via power control [8], orthogonal signals [9] spatial diversity [10], or interference alignment [11]. On the contrary, in this paper we argue that near-far scenarios are in fact a resource that can be leveraged to improve the performance of UWANs, e.g., via multipacket reception techniques using successive interference cancellation. Yet, as we show in our field experiment, even when interference cancellation is limited or unavailable, since transmissions from a farther node do not substantially affect the reception of signals from a closer node, the two nodes can still be allowed to transmit simultaneously, as long as the destinations of the two nodes are different.

Allowing transmissions of near-far node pairs (NFNPs) to different destination nodes in a spatial-reuse TDMA fashion opens the possibility to overcome one of the most limiting assumptions in scheduling UWANs, namely, that the network can support the transmission of only a single packet type. Specifically, while most existing channel access protocols allocate transmissions considering either contention-based communications (e.g., Carrier-Sense Multiple Access-CSMA, and Multiple Access Collision-Avoidance-MACA) or contentionfree communications (e.g., TDMA and spatial-reuse TDMA), opportunistic transmissions of NFNPs allow different communications patterns to co-exist. In particular, the messages that are not subject to stringent delivery constraints can be allocated for opportunistic transmissions, so that they do not have to wait for pre-assigned slots; similarly, scheduled transmissions with tighter constraints can co-exist with opportunistic traffic without being harmed by the latter.

In this paper, we describe a scheduling MAC algorithm for both contention-free and opportunistic transmissions. Our algorithm, referred to as the near-far spatial reuse TDMA (NFTDMA), maximizes the network throughput and minimizes the delivery delay by allowing multiple nodes to transmit in the same time slot. To that end, given information on the network topology and the NFNPs (e.g., provided by an initial topology discovery phase), we formulate an optimization problem that yields collision-free scheduling for a target minimum packet transmission rate. Moreover, we allow each transmitting node to locally choose the best communication type for each time slot, and provide it with guidelines to prioritize transmissions.

To the best of our knowledge, together with our recent work [12] about leveraging on the near-far effect in handshakebased MAC protocols, our NF-TDMA algorithm is the first attempt to exploit the near-far effect to achieve a performance improvement in underwater acoustic networks, and the first attempt to explicitly consider this phenomenon in an optimized scheduling protocol. We prove our argument both in simulations and in a sea experiment, in order to show the practicality of our approach. Our contribution is three-fold:

1) A method to exploit the near-far effect to increase the network throughput, even when all nodes are directly connected to the same sink and spatial reuse is seemingly not possible, and even without interference cancellation capabilities;

2) A scheduling algorithm to service both contention-free and opportunistic communications, each having different objectives;

3) A combination of a centralized schedule with a distributed one to obtain an optimal channel utilization for a given interference cancellation capability of the system.

We compare our results against those achieved by a basic TDMA protocol, as this is the solution all currently available collision-free spatial-reuse TDMA schemes would fall back to when all nodes are directly connected to a single sink. Our results show that our NF-TDMA achieves much better throughput and delivery delay. This comes at a slight cost in terms of fairness in opportunistic transmission chances. These results are verified and our assumptions are validated in a field experiment, where we demonstrate our algorithm in real time using off-the-shelf acoustic modems.

The remainder of this paper is organized as follows. In Section II we survey relevant related work in the area. Section III introduces the system model along with some assumptions and preliminary definitions of the relevant network metrics considered in this paper. Section IV proposes our NF-TDMA protocol and the algorithm to obtain a scheduling solution given a network topology. Section $\mathrm{V}$ shows preliminary simulation results, which are instrumental to proving the superior capabilities of our protocol with respect to plain TDMA before testing our solution in a field experiment (Section VI). Finally, we offer some concluding remarks in Section VII.

\section{StATE-OF-THE-ART}

To compensate for the low channel utilization of TDMA, UWAN-MAC [13] proposes to schedule sleep/transmit/receive epochs among the nodes via a network discovery mechanism, and to adaptively shift these epochs over time in case joining nodes cause receive-receive collisions. Similarly, I-TDMA [14] proposes to postpone colliding transmission schedules by assuming that the propagation delay is known (something which was not strictly needed in UWAN-MAC). However, these solutions are prone to uncontrolled drifts in 
the sleeping schedules of the nodes [15]. The authors in [16] propose a centralized and a distributed scheduling scheme. The former prioritizes the nodes that need to occupy the channel for a shorter amount of time. The latter is based on clustering and on centralized scheduling in each cluster. Clustering is employed also in [17], where a hierarchy of two nested TDMA schedules is proposed: the outer one is used for surface radio/acoustic gateway stations, whereas the inner one is used by underwater nodes. Mobility is supported by reserving a pre-allocated slot for opportunistic cluster membership packets. In [18], it is proposed to distribute 2hop topology information and to schedule nodes based on their ID, by adapting the TDMA frame length to the transmission requirements, whereas [19] bases its scheduling choices on a priority list known a priori and on a tight scheduling of the nodes without guard times in each slot. The authors in [20] take a different approach by assuming that time is slotted and that each node in a single broadcast domain network can transmit in any slot with a given probability. A linear program is solved to compute the optimal transmission probabilities for all nodes. The advantage of this approach over a uniform transmission probability assignment is shown to increase with increasing packet size. Under the same system assumptions, the approach in [21] also proposes heuristic policies based on the exchange of neighboring node counts. A hybrid protocol that alternates TDMA-scheduled slots and unscheduled slots is proposed in [22], where the slot length also includes the maximum propagation delay.

Exploiting the propagation delay to avoid receiver-side collisions has been shown to be a promising approach in [23]. The STUMP protocol [24] extends this approach by scheduling transmissions in a multihop network so that all types of primary conflicts can be avoided. The design assumes that the nodes are aware of propagation delays and transmission requirements in their 2-hop neighborhood. STUMP-WR [25] adds routing to the picture, which operates on links instead of rings and therefore requires only the solution of a simpler link scheduling problem. The ST-MAC protocol [26] solves a similar space-time scheduling problem by avoiding conflicts and including a capture model. The optimal solution is compared to a simpler heuristic where the node whose transmission would be completed last is scheduled first. DOS [27] further extends ST-MAC with an on-demand mechanism to update transmission schedules over a hierarchical topology. The approach is shown to outperform ST-MAC when the latter is re-designed to improve fairness. The approach in [28] introduces a time-evolving conflict graph which is exploited by centralized algorithms to schedule transmissions under different cost functions, leading to fair, traffic-based or mostheavily-loaded-first scheduling. Scheduling in the broader context of a converge-casting network was considered in [29], [30], [31]. In particular, [29] presents a Receiver-Oriented Sleep Scheduling (ROSS) protocol, where the network is designed to operate data fusion. Sibling nodes on the convergecasting tree coordinate with their parent nodes in case of scheduling conflicts, and the coordination process is allowed to escalate upstream until the conflict is resolved. Similarly, [30] organizes the scheduling of transmissions over the converge- casting tree tier by tier, in a way that reduces the latency of the aggregation process. A number of combinations between scheduling and routing policies are compared in [31], where the best performance was yielded by fair scheduling coupled with a relay selection policy that favors the less interfered node.

The work in [32] takes a fundamental approach by showing that optimal schedules in any network with a single broadcast domain are status, and that the maximum achievable throughput is $N / 2$, where $N$ is the number of nodes. The authors provide a computationally efficient algorithm to obtain good schedules. The same result has been shown to extend to complex topologies in [33]. Based on the above work, [34] adds realistic modem constraints and shows that an implementation of the scheme proposed in [32] actually works in practice in simple topologies. The approach in [7] further observes that any scheduling approach which relies on topology and propagation delay information may incur excessive overhead or even fail when such information is subject to change due to even limited mobility or channel variability. For this reason, a topology-transparent schedule is taken as the basis to create a topology-based schedule which is also robust to topological changes. The results are successfully tested both in simulations and in a field experiment. In [12], we describe a heuristic method to limit the exposed terminal problem by allowing near and far nodes to engage in simultaneous communication sessions to different destinations. However, this approach was tailored to handshake-based communications, and is not suitable for networks with heavy traffic requirements such as those considered here.

Unlike all previous approaches, in this paper we argue that not all collisions are harmful, and that near-far communication scenarios can be in fact exploited to decouple interfering transmissions. As we show in our field experiment, such opportunity is available even when the system does not have interference cancellation capabilities. This goes to the benefit of network performance, as a spatial reuse algorithm can afford to schedule transmissions more often, on average. With the above in mind, we propose a scheduling algorithm that is specifically designed to exploit near-far transmission opportunities. Additionally, our algorithm supports the management of packets of different types, with different service constraints. We introduce our scheduling design by starting from some preliminary definitions in the next section.

\section{System ModeL}

In this section, we introduce the system model and the objectives for resource allocation considered in this work. Our system includes a group of nodes (e.g., divers or submerged devices) represented by a set $\mathcal{N}=\left\{i_{1}, \ldots, i_{N-1}\right\}$ of nodes. Each of the nodes is directly connected to a single node, $i_{0}$, referred to as the cluster head, or sink. However, a node $i_{n} \in \mathcal{N}$ may or may not be connected to a node $i_{m} \in \mathcal{N}, m \neq n$. Each node in $\mathcal{N}$ is assumed to always have a status packet to transmit to node $i_{0}$, and occasionally may also have an opportunistic packet to transmit to one of its one-hop neighbor nodes (including node $i_{0}$ ). Status packets convey the status of 
the node (location, energy level, air supply, mission progress, etc.) and are to be transmitted in a contention-free manner. In addition, status packets are transmitted and should be received by the cluster head at least once every $T_{L}$ seconds. On the contrary, the transmission of opportunistic packets takes place randomly. Since we aim to reduce the transmission delay of opportunistic packets, as long as the minimal rate for the transmission of status packets is maintained, the transmission of opportunistic packets is preferred. All nodes are allowed to send opportunistic packets to any node, whereas status packets are transmitted by all nodes except the cluster head, which is instead the common destination. A node is assumed to always have a status packet to transmit, whereas opportunistic packets are less frequent.

\section{A. Problem Definition}

We are interested in a collision-free TDMA-based periodic transmission schedule for each node $i_{n} \in \mathcal{N}$ with respect to the cluster head. The time frame of this schedule consists of time slots allocated to nodes for transmissions. The time frame is set by the cluster head node, $i_{0}$, which in turn broadcasts the solution to its one-hop neighbors, i.e., to the nodes in $\mathcal{N}$. While this is a centralized solution, the special position of node $i_{0}$ ensures fast and reliable sharing of the scheduling solution with all nodes. To ensure that the network can easily adapt to the motion of nodes, we target a scheduling solution with low communication overhead. The nodes are assumed to only evaluate their one-hop neighbor list and share it with the sink node. An example of a process to obtain this information is presented in [35], where nodes transmit in a pre-determined manner during the network setup phase, such that by receiving packets, a node can build its own one-hop list. Given onehop information, the sink node evaluates the network topology and replies only with the schedule. Moreover, we allow all nodes to locally decide which packet type (i.e., either status or opportunistic) to transmit. That is, the nodes can maintain their own packet queue and service it locally. With respect to the network performance, we are interested in maximizing the network throughput, minimizing the transmission delay, and obtaining high network fairness. In the following we define these objectives.

1) Throughput-Status Packets: Let us assume that a status packet consists of $N_{\text {bit }}$ bits. Also, call $x_{n}^{\mathrm{s}}$ the number of successfully received status packets sent by node $i_{n}$ to node $i_{0}$ over a given time interval of duration $T$ seconds. Since every node always has a status packet to transmit to node $i_{0}$, the network throughput of status packets is defined as

$$
\rho_{\text {through }, \mathrm{s}}=\frac{1}{T} \sum_{n=1}^{N-1} x_{n}^{\mathrm{s}} N_{\mathrm{bit}} .
$$

Let $t_{n, j}^{\mathrm{s}}$ be the time when the $j$ th status packet of node $i_{n}$ is received by the sink. Considering the objective of receiving status packets at least once every $T_{L}$ seconds, we maximize $\rho_{\text {through,s }}$ under the constraint that

$$
t_{n, j+1}^{\mathrm{s}}-t_{n, j}^{\mathrm{s}}<T_{L}, \forall n, j .
$$

In this work we do not directly optimize the fairness in scheduling status packets. Instead, we consider a minimum fairness of status packets by defining a vector $\mathbf{c}$ of minimal number of receptions over one time frame of the schedule. ${ }^{1}$

$$
x_{n, 0}^{\mathrm{s}} \geq c_{n}, \forall n .
$$

We choose $c_{n} \geq 2, \forall n$, such that each node is allocated at least two packets to transmit. Hence, as long as the time frame of the schedule is smaller than $T_{L}$, a node can allocate both status and opportunistic packets in the same time frame.

2) Scheduling Delay - opportunistic packets: Define $x_{n, m}^{\mathrm{o}}$ as the number of opportunistic packets generated by node $i_{n}$ and successfully received by node $i_{m}$, and define a set $\mathcal{M}_{n}$ such that $m \in \mathcal{M}_{n}$ if and only if $x_{n, m}^{\mathrm{o}}>0$. Also let $t_{n, m, j}^{\mathrm{o}}$ be the delay from the time an opportunistic packet $j$ is transferred to the MAC layer of source $i_{n}$ until it is successfully delivered to its destination $i_{m}$. That is, $t_{n, m, j}^{\mathrm{O}}$ captures both the end-toend transmission delay and the queuing delay. The average per-node scheduling delay of opportunistic packets is defined as (recall that the cluster head can also send opportunistic packets)

$$
\rho_{\text {delay }, \mathrm{o}}=\frac{1}{N} \sum_{n=0}^{N-1} \frac{1}{\left|\mathcal{M}_{n}\right|} \sum_{m \in \mathcal{M}_{n}} \frac{1}{x_{n, m}^{\mathrm{o}}} \sum_{j=1}^{x_{n, m}^{\mathrm{o}}} t_{n, m, j}^{\mathrm{o}} .
$$

3) Fairness - opportunistic packets: We measure fairness by comparing the differences in the per-node throughput of the opportunistic packets. By applying Jain's fairness index [36], we define the throughput fairness of opportunistic packets to be

$$
\rho_{\text {fair }, \mathrm{o}}=\frac{\left(\sum_{n=0}^{N-1} \sum_{m=0, m \neq n}^{N-1} x_{n, m}^{\mathrm{o}}\right)^{2}}{N \sum_{n=0}^{N-1}\left(\sum_{m=0, m \neq n}^{N-1} x_{n, m}^{\mathrm{o}}\right)^{2}} .
$$

\section{B. Topology Information}

With respect to the cluster head, $i_{0}$, the information about the receiver-side topology is given in the form of an $(N-$ $1) \times(N-1)$ matrix M. Specifically, the diagonal elements of $\mathbf{M}$ represent the direct links between node $i_{0}$ and its neighbor nodes, while the rest of the matrix entries indicate the possibility of receiving in the presence of interference from another node. More specifically, the $(x, y)$ th entry, $M_{x, y}$, equals 1 if node $i_{0}$ can successfully receive a packet from node $i_{x}$ even while node $i_{y}$ is transmitting, and 0 otherwise. ${ }^{2}$. To form $\mathbf{M}$, we require a probability matrix $\mathbf{P}$ whose entry $P_{x, y}$ represents the probability of successful reception of packets from node $x$ while node $y$ is transmitting. Then, $M_{x, y}=1$ if $P_{x, y} \geq \theta$, where $\theta$ is a target packet reception probability. Both matrices $\mathbf{M}$ and $\mathbf{P}$ are inputs to our algorithm, and can

\footnotetext{
${ }^{1}$ Throughout this paper, we indicate vectors via bold lower-case letters (e.g., a), matrices via bold upper-case letters (e.g., A), and matrix/vector entries via indices subscripted to the non-bold version of the same matrix/vector name (e.g., $a_{j}, A_{x, y}$ ).

${ }^{2}$ We assume that the cluster head only has information about NFNPs. The event that more than two nodes transmit simultaneously is still possible, and managed as explained in Section IV-B
} 
be measured during an initial phase for topology discovery. ${ }^{3}$ During the same process, the nodes in $\mathcal{N}$ obtain their list of one-hop neighbor nodes and share it with the cluster head. As a result, the cluster head is able to evaluate the network topology.

In case $M_{x, y}=1$ and $M_{y, x}=1$, nodes $i_{x}$ and $i_{y}$ can be scheduled for simultaneous transmissions, as neither would impede the reception of the other. However, in case $M_{x, y}=1$ but $M_{y, x}=0$, if transmitting together, node $i_{x}$ will overshadow (or jam) the transmissions of node $i_{y}$, i.e., the near-far effect occurs. Note that the former case is only possible when the receiver holds multiple packet reception capabilities or applies interference cancellation techniques. However, the latter case does not involve interference cancellation techniques. An example of a topology exhibiting a nearfar scenario is illustrated in Fig. 2a. In this example, node $i_{1}$ is much closer to node $i_{0}$ than node $i_{2}$ and jams the transmissions of the latter. In this paper, we specifically assume that there is at least one near-far scenario across the network.

\section{The NeAR-FAR Scheduling Solution}

In this section, we discuss in detail our spatial reuse scheduling algorithm that exploits the near far effect. We start by describing the key idea behind our solution, and then present our algorithm. We discuss the details of forming the scheduling TDMA frame, how to determine the types of packets transmitted, and how to set the list of destinations of opportunistic packets. For a clearer description of our algorithm, throughout the following subsections we refer stepby-step to the example given in Fig. 2a. There, the clusterhead $i_{0}$ has to compute a schedule for all its connected nodes $i_{1}, \ldots, i_{4}$, where some nodes are subject to the nearfar effect: for example, a transmission by node $i_{2}$ would be shadowed by a transmission from $i_{1}$, if the two corresponding signals are received at the same time. The following subsection highlights the key idea and operation of our algorithm, whereas Section IV-B describes the algorithm's details.

\section{A. Key Idea}

Our solution is based on the observation that in a near-far situation, only one collision occurs and the jammer can still transmit. Then, the receiver can directly decode the packet from the jammer while applying interference cancellation techniques to decode the jammed packet. In that sense, our solution is similar to cognitive radio schemes, where secondary nodes are allowed to transmit in the band of primary nodes. Since interference cancellation is not always feasible, we also allocate transmission epochs where the reception of packets of each node is guaranteed. In these transmission epochs, we allow the jammer to transmit only if it employs power control to ensure the reception of the transmission from the jammed node. Moreover, as we demonstrate in our field experiment, even without interference cancellation capabilities,

\footnotetext{
${ }^{3}$ Topology information can be obtained by measuring the rate of successful packets [37], [38] or by estimating the SINR through measuring the distances among the nodes and applying an attenuation model [6]. This process is beyond the scope of this paper.
}

spatial reuse is still possible by utilizing information about NFNPs and guiding the jammer and jammed nodes to transmit packets to different destinations.

Our NF-TDMA algorithm is unique in the sense that although (like any other TDMA scheduling algorithm) it is designed for the contention-free communication of status packets, it also serves the transmission of opportunistic packets. To that end, while the basic schedule is set by the cluster head, nodes can distributedly decide whether to transmit a status packet or an opportunistic packet. This is determined while satisfying a minimum transmission rate of status packets and depending on opportunistic packet arrivals. To avoid collisions, in the case of opportunistic packets, a node decides upon the destination node from a list of possible candidates offered by the cluster head already at the initial stage when the scheduling solution is derived.

We expect the near-far situation to persist even when the receiver, jammer, and jammed nodes slowly move, which is the common case in UWANs. This is motivated by the results in [39] which shows that there is a strict boundary between the cases of low and sufficient signal-to-interference ratio (SIR) to correctly receive a packet belonging to a NFNP. This implies that exploiting the near-far effect in spatial reuse scheduling algorithms does not strictly require frequent topology and schedule updates.

\section{B. NF-TDMA}

The output of the NF-TDMA scheduling algorithm is an $N \times L$ matrix $\mathbf{S}$ to allocate the transmissions of $N$ nodes over $L$ time slots. Given $\mathbf{S}$, a node $i_{n}$ is allowed to transmit in time slot $\ell$ with probability $S_{n, \ell}$. Then, the case of $\sum_{n=0}^{N-1} S_{n, \ell}>1$ for some $\ell$ (i.e., more than one nodes are allowed to transmit together in one or more slots) is typically referred to as spatial-reuse TDMA, and the case where $\exists i_{m}, i_{n}, \ell \quad \mid S_{n, \ell}>0, S_{m, \ell}>0, M_{m, n} \neq M_{n, m}$ characterizes NF-TDMA. The latter case means that between two nodes that transmit in a given slot, only one can be received (say only $m$ ). In the following, we describe the procedure to obtain $\mathbf{S}$. The steps of the algorithm are listed in Algorithm 1, Fig. 2b. Our implementation of the algorithm is published for reproducibility. ${ }^{4}$

1) Preliminary Steps: Before obtaining the schedule, the cluster head $i_{0}$ performs three preliminary steps to rearrange the topology information in matrix $\mathbf{M}$ towards scheduling transmissions. First, node $i_{0}$ constructs a list $\mathcal{R}$ of all node pairs involved in a near-far situation. This list is found by inspecting non-symmetric entries in matrix $\mathbf{M}$. Referring to the example in Fig. $2 \mathrm{a}$, these would be nodes $\left(i_{2}, i_{1}\right)$. To formalize this,

$$
\left(i_{n}, i_{m}\right) \in \mathcal{R} \quad \text { if } M_{n, m}=0 \text { and } M_{m, n}=1,
$$

where the second node $i_{m}$ is the jammer node (see lines 1-4 in Algorithm 1, Fig. 2b).

For each far node $i_{n} \in \mathcal{R}$ and its near peer $i_{m}$, node $i_{0}$ is able to estimate an interference cancellation probability, $p_{n, m}$.

\footnotetext{
${ }^{4}$ http://marsci.haifa.ac.il/share/diamant/NearFarPublishCode.zip
} 


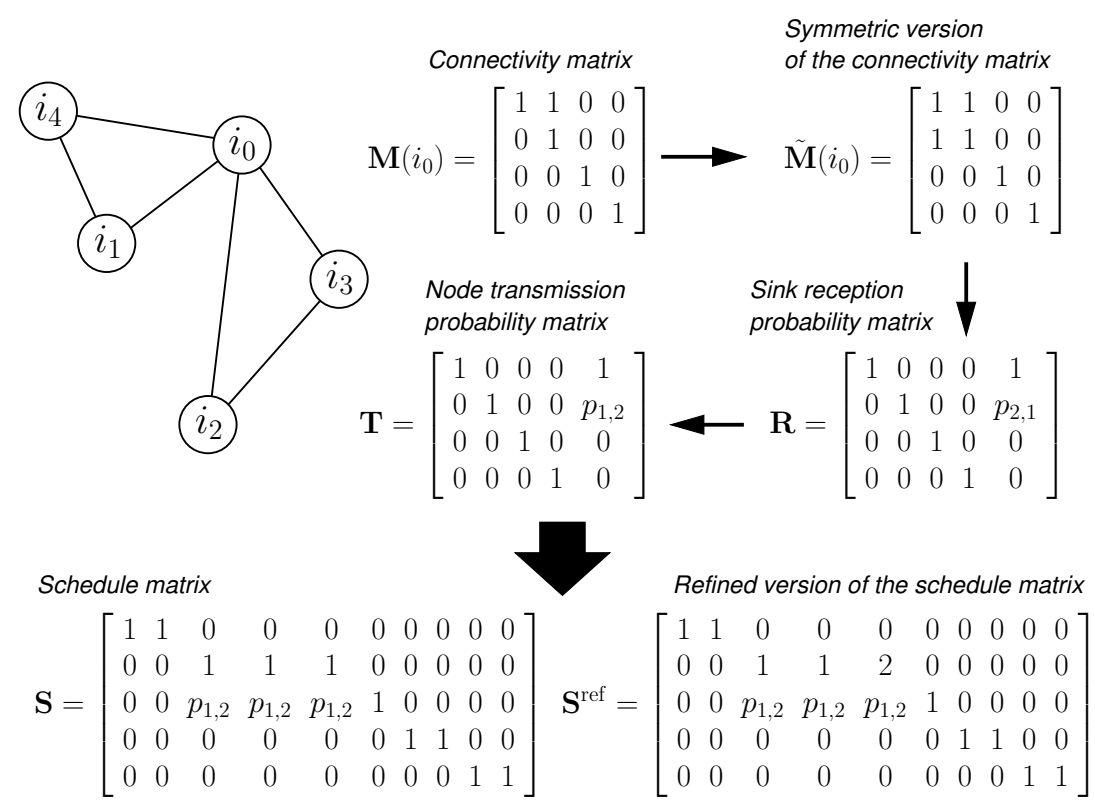

(a)

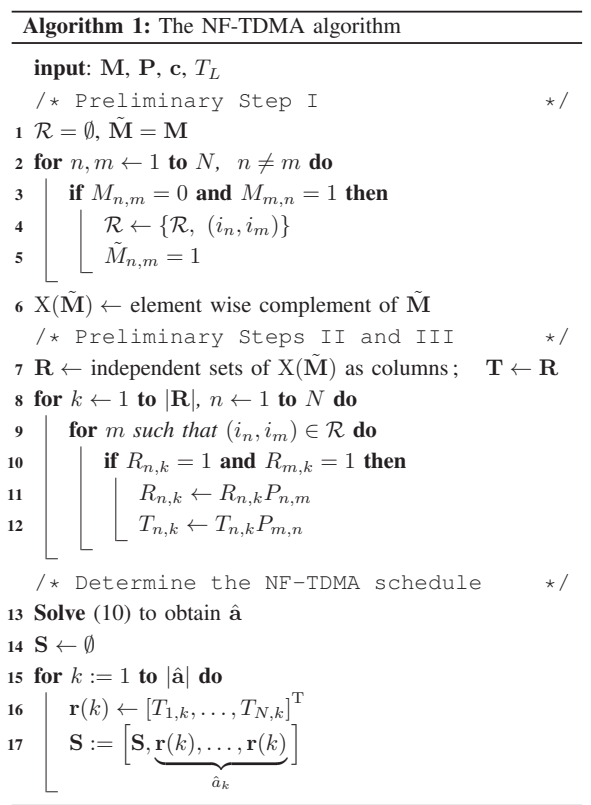

(b)

Fig. 2. (a) Example for the illustration of the NF-TDMA algorithm. (b) Pseudocode of our proposed NF-TDMA algorithm.

The latter is the probability of properly decoding a packet from $i_{n}$ while simultaneously receiving a packet from node $i_{m} .{ }^{5}$ Note that if $i_{m}$ is the jammer, then $p_{n, m}<p_{m, n}$. Clearly, $p_{n, m}$ is a function of the SNR and of the superposition of the packets. Instead of avoiding the near-far effect, we exploit it by allowing nodes $i_{n}$ and $i_{m}$ to transmit simultaneously. For this, we "fill up the gaps" and create a symmetric version of M, namely $\tilde{\mathbf{M}}$, such that

$$
\tilde{M}_{n, m}= \begin{cases}1 & , \quad\left(i_{n}, i_{m}\right) \in \mathcal{R} \\ M_{n, m} & , \text { otherwise }\end{cases}
$$

(see line 5 in Fig. 2b). Matrix $\tilde{\mathbf{M}}$ for our example is given in Fig. 2a.

Second, we find all the possible collision-free transmission scheduling solutions in the network. For this, we form the symmetric matrix $\mathrm{X}(\tilde{\mathbf{M}})$, where $\mathrm{X}(\cdot)$ denotes the element-wise 1 -complement operator. In case entry $\mathrm{X}(\tilde{\mathbf{M}})_{i, j}>0$, then both nodes $i$ and $j$ may transmit together. Next, we convert $\mathrm{X}(\tilde{\mathbf{M}})$ into a connected graph, where nodes are vertices, and non-zero entries are edges. The list of all independent sets of this graph (see also [41]), $\mathbf{w}=\left\{\mathbf{w}^{(1)}, \ldots, \mathbf{w}^{(K)}\right\}$, represents all the combinations of nodes that can transmit together. To find the best possible collision-free transmission scheduling solution in the network, our goal is to choose those independent sets from $\mathbf{w}$ that lead to the maximum link utilization while guaranteeing the minimal required per-node transmission rate. These independent sets will then form the TDMA schedule time frame. Consider for example that $\hat{K}$ independent sets are chosen from $\mathbf{w}$. Then, the schedule time frame consists of $\hat{K}$ time slots such that in the $k$ th time slot, only nodes that are included in $\mathbf{w}^{(k)}, k=1, \ldots, \hat{K}$ can transmit. Each entry

\footnotetext{
${ }^{5}$ The interference cancellation probability can be found by calculating or measuring the signal-to-interference-pulse-noise ratio (SINR) for each of the received symbols and setting a threshold for the target symbol error rate probability (e.g., see [40])
}

$n, n=1, \ldots, N-1$ in an independent set $\mathbf{w}^{(k)}$ can have the following three values:

1) $w_{n}^{(k)}=0$ : node $i_{n}$ can not transmit;

2) $w_{n}^{(k)}=1$ : node $n$ can transmit, and the reception of its packet will not be affected by interference with probability 1 ;

3) $w_{n}^{(k)}=p_{n, k}$ : node $n$ can transmit, and the reception of its packet will not be affected by interference with probability $0<p_{n, k}<1$.

Note that the third case can occur when there is another node, $i_{m}$, who forms a near-far pair with node $i_{n}$ and for which $w_{n}^{(k)}>0$. That is, the third case applies when

$$
\exists i_{m} \mid\left(i_{n}, i_{m}\right) \in \mathcal{R} \wedge w_{m}^{(k)}>0 .
$$

In this case, we set $p_{n, k}=p_{n, m}$. Clearly, without interference cancellation capabilities we have $p_{n, k}=0$. As a first-order approximation, near-far scenarios with a jammed node and more than one jammer nodes are considered by setting $\left(1-p_{n, k}\right)$ as a multiplication of all the relevant near-far error probabilities. For example, if node $i_{n}$ is the "far" node with respect to both nodes $i_{m}$ and $i_{q}$, and $w_{m}^{(k)}>0, w_{q}^{(k)}>0, w_{n}^{(k)}>0$, we set $p_{n, k}=1-\left(1-p_{n, m}\right)\left(1-p_{n, q}\right)$. The $K$ different vectors $\mathbf{w}$ are arranged in columns to form an $(N-1) \times K$ sink reception probability matrix $\mathbf{R}$, whose entry $R_{n, k}$ is the probability that the cluster head node $i_{0}$ receives a packet from node $i_{n}$ for the $k$ th transmission set (see lines 7-11 in Fig. 2b). Matrix $\mathbf{R}$ for our example is presented in Fig. 2 a.

Third, we form an $(N-1) \times K$ node transmission probability matrix $\mathbf{T}$, whose entry $T_{n, k}$ represents the probability that node $i_{n}$ transmits in the $k$ th possible transmission combination. For a node $i_{n}$ for which $w_{n}^{(k)}=1$, we set $T_{n, k}=1$. However, for a node pair $\left(i_{n}, i_{m}\right) \in \mathcal{R}$ and time slot $k$ for which $w_{n}^{(k)}>0$ and $w_{m}^{(k)}>0$, we prefer to allow the jammer node $i_{m}$ to always transmit. In this case, the probability that 
the jammed node $i_{n}$ transmits depends on the ability of $i_{0}$ to receive the jammer (rather than the jammed node). Hence, we set $T_{m, k}=1$ and $T_{n, k}=p_{m, n}$. As will become clear in the next section, this preference is because when probability $p_{n, m}$ is low, our scheduling solution would allocate at least one exclusive transmission slot to the jammed node. When this happens, the jammer would be allowed to actually transmit along with its jammed node pair only when it employs power control. For our example, matrix $\mathbf{T}$ is shown in Fig. 2a.

2) Forming the Schedule: The stage is now set to present our NF-TDMA algorithm to obtain the scheduling matrix $\mathbf{S}$. We observe that $\rho_{\text {through,s }}$ and $\rho_{\text {delay,o }}$ in (1) and in (4), respectively, can be optimized by maximizing the channel utilization, which corresponds to maximizing the number of collision-free transmissions. That is because both the number of received packets and the time a packet is delayed in transmission queues are affected by the number of collision-free transmissions. Hence, in the setting of spatial-reuse TDMA, the schedule converges to allocating the maximum possible number of transmission time slots while ensuring that packets arrive without collisions. To that end, we denote a $K \times 1$ vector a, whose entries $a_{j}$ represent the number of times column $j$ from $\mathbf{T}$ is chosen in the scheduling solution. In matrix form, we obtain the reception vector

$$
\mathbf{r}(\mathbf{R}, \mathbf{a})=\mathbf{R a},
$$

such that for row $n$ in matrix $\mathbf{R}, r_{n}(\mathbf{R}, \mathbf{a})$ packets are sent by node $i_{n}$ and successfully received at node $i_{0}$.

To allow a minimum number of transmissions (including at least one status packet) by each node $i$, we fix the number of time slots in one time frame to be

$$
L=\frac{T_{L} \cdot \max _{i} c_{i}}{T_{s}},
$$

where $T_{s}$ is the duration of the time slot. Then, considering the scheduling constraints in (2) and (3), the scheduling problem can be written as

$$
\begin{gathered}
\hat{\mathbf{a}}=\underset{\mathbf{a}}{\operatorname{argmax}} \sum_{n=1}^{N-1} r_{n}(\mathbf{R}, \mathbf{a}) \\
\text { s.t. } \quad \sum_{n} a_{n}=L, \\
\quad r_{n} \geq c_{n}, \forall n \in \mathcal{N} .
\end{gathered}
$$

Note that by (5), the goal in fairness is to equalize the transmission opportunities of nodes. While we do not explicitly optimize fairness in (10), we relate to the fairness by imposing constraint (10c) to satisfy requirement (3). This way, a node $i_{n}$ gets a minimum of $c_{n}$ contention-free transmission slots per frame, which ensures some fairness in the transmission of status packets. However, the fairness of opportunistic packets is not considered. Problem (10) is an NP-hard integer linear program, whose worst-case complexity grows exponentially with the size of a. However, as shown in [42], [43], it can be solved in polynomial time (on average) via the branch-andbound algorithm.

The solution â from (10) is readily used to find the schedule matrix $\mathbf{S}$. Let $\mathbf{T}_{j}$ represent the $j$ th column of the node transmission probability matrix $\mathbf{T}$, and $\hat{a}_{j}$ be the $j$ th element of â. We have

$$
\mathbf{S}=[\underbrace{\mathbf{T}_{1}, \ldots, \mathbf{T}_{1}}_{\hat{a}_{1}}, \underbrace{\mathbf{T}_{2}, \ldots, \mathbf{T}_{2}}_{\hat{a}_{2}}, \ldots, \underbrace{\mathbf{T}_{K}, \ldots, \mathbf{T}_{K}}_{\hat{a}_{K}},],
$$

i.e., the $j$ th column of $\mathbf{T}$ is replicated $\hat{a}_{j}$ times. Since our objective is to maximize the network throughput, the solution for (10) and the definition in (11) are set by considering matrix $\mathbf{R}$, i.e., the packet reception requirement rather than the packet transmission requirement. In turn, schedule $\mathbf{S}$ indicates which node can transmit in each time slot and the probability that it will actually transmit. Hence, the solution of (10) is readily transformed into the scheduling matrix $\mathbf{S}$, whose columns are replicas of the columns of the node transmission probability matrix T.Therefore, the scheduling solution matrix $\mathbf{S}$ contains $\hat{a}_{k}$ replicas of $\mathbf{T}_{k}$ (see lines 13-17 in Fig. 2b). In Fig. 2a, we show the resulting scheduling solution $\mathbf{S}$ for our example, where we consider $L=10, c_{n}=2 \forall n$, see (10c), $p_{1,2}=1$, and $p_{2,1}=0.3$. Here, entry $S_{n, \ell}$ is the probability that node $i_{n}$ transmits in slot $\ell$.

3) Determining the Packet Type: In each time slot, $t$, a node scheduled to transmit can send either a status packet or an opportunistic packet. Considering the need to send status packets, opportunistic packets are transmitted only if there is a high probability to transmit a status packet in the next few time slots such that constraint (2) is met. To formalize this, let $t_{m}, m=1,2, \ldots$ be a future time slot index such that $t_{m}>t$ and $S_{n, t_{m}}>0$. Also denote as $\mathcal{E}_{n, t, t_{m}}$ the event for which node $i_{n}$ will transmit between the current time slot $t$ and a future time slot $t_{m}$. The probability that event $\mathcal{E}_{n, t, t_{m}}$ occurs is

$$
\mathbb{P}\left[\mathcal{E}_{n, t, t_{m}}\right]=1-\prod_{i=1}^{m}\left(1-S_{n, t_{i}}\right)
$$

Let $\hat{m}$ be the smallest subindex for which $\mathbb{P}\left[\mathcal{E}_{n, t, t_{m}}\right]>\Theta_{\mathrm{Tx}}$, where $\Theta_{\mathrm{Tx}}$ is a fixed threshold. Then, node $i_{n}$ will transmit an opportunistic packet in time slot $t$ if there is an opportunistic packet in the queue, and if the time elapsed from the previous time slot where node $i_{n}$ transmitted a status packet (say, $t_{\ell}$ ) until the future time slot $t_{\hat{m}}$ is smaller than the required value $T_{L}$, see (2). Formally, if $\exists \hat{m}$ such that

$$
t_{\hat{m}} \cdot T_{s}+T^{\max }-t_{\ell} \cdot T_{s}<T_{L}
$$

where $T^{\max }$ is the maximum propagation delay in the network. Otherwise, a status packet is transmitted. The condition in (13) makes sure that the time elapsed between transmissions of status packets would not exceed $T_{L}$ seconds. However, no consideration is given to the case where fewer transmission slots than opportunistic packets are available. In the latter case, opportunistic packets are queued up, and transmitted in a first-come-first-served fashion. The delay induced by this event is considered in the definition of the scheduling delay metric in (4), and is analyzed in our numerical results and field experiment.

4) Refinement: Note that the utility function in (10) considers the packet reception probability, while the scheduling solution sets the transmission probability of the nodes. As we 
mentioned above, in case the $k$ th transmission set is chosen multiple times (i.e., $\hat{a}_{k}$ is large), this allows the reception of at least $c_{n}$ packets for a node $i_{n}$ whose reception probability $p_{n, k}$ at the cluster head is low. That is, the solution in $\mathbf{S}$ may avoid some simultaneous transmissions by the near-far nodes $i_{m}$ and $i_{n}$ if these transmissions may reduce the chances of the jammed node $i_{n}$ to be received below its required flow constraints. This comes directly from the solution of (10) by choosing $\hat{\mathbf{a}}_{k^{\star}} \geq 1$ for at least one transmission set $k^{\star}$ in which node $i_{n}$ can be received without interference. However, these avoided simultaneous transmissions can still be allowed if the jammer $i_{m}$ reduces its transmission power such that although $i_{n}$ and $i_{m}$ simultaneously transmit, the packets of $i_{n}$ are still received. Hence, considering a node pair $\left(i_{n}, i_{m}\right) \in \mathcal{R}$, for which only node $i_{n}$ is scheduled to transmit in set $k^{\star}$, we observe that a refinement is possible.

To further improve the network throughput, we allow the jammer node $i_{m}$ to transmit in set $k^{\star}$ only if it employs power control. Such power control should still ensure the reception of node $i_{n}$. Let $P_{n}$ be the reception power of a packet from a node $i_{n}$ at the cluster head $i_{0}$, such that the power ratio between the jammed packet and the jamming packet is

$$
\rho_{P}(n, m)=\frac{P_{n}}{P_{m}} .
$$

It follows that to transmit in set $k^{\star}$, node $i_{m}$ must reduce its power by a factor $\rho_{P}^{2}(n, m)$. This will lead to an inverse nearfar scenario where node $i_{n}$ becomes the jammer and node $i_{m}$ becomes the jammed node.

After obtaining solution $\mathbf{S}$ through (10), we performed a refinement by identifying all the transmission sets $k^{\star}$ according to the following procedure $\forall\left(i_{n}, i_{m}\right) \in \mathcal{R}$. The following algorithm identifies the transmission sets $k^{\star}$ in $\mathbf{S}$ in which near-far simultaneous transmissions are avoided, and mark them in a new refined scheduling matrix $\mathbf{S}^{\text {ref }}$ :

1) Initialize $\mathbf{S}^{\text {ref }}=\mathbf{S}$.

2) Form a matrix $\tilde{\mathbf{R}}$ of all maximal independent sets of the symmetric matrix $\tilde{\mathbf{M}}$ (see (7)).

3) Identify a column $k^{\star}$ in $\mathbf{S}$ not included in $\tilde{\mathbf{R}}$ such that $S_{n, k^{\star}}=1$ and $S_{m, k^{\star}}=0$.

4) Make an indication $S_{m, k^{\star}}^{\mathrm{ref}}=n$.

To execute the refined NF-TDMA algorithm, instead of $\mathbf{S}$, the sink node communicate $\mathbf{S}^{\text {ref }}$ to the other nodes. Then, a node $i_{m}$ for which $S_{m, k^{\star}}^{\text {ref }}>1$ is allowed to transmit in time slot $k^{\star}$ only if it reduces its transmission power by a factor $\rho_{P}^{2}(n, m)$ from (14). In Fig. $2 \mathrm{a}$, we denoted $S_{1,5}^{\text {ref }}=2$. This means that node $i_{1}$ is allowed to transmit in the 5 th time slot only if it reduces its transmission power by a factor $\rho_{P}^{2}(2,1)$. Naturally, power control can be performed only if $i_{m}$ can locally calculate factor (14) or is explicitly told which power to use, and thus this refinement may not always be performed.

5) Destination of opportunistic packets: While nodes make the decision whether to transmit a status packet or an opportunistic packet distributedly, they can be guided to choose the destination node of their packets properly. Since the cluster head is aware of the connectivity list of each of its one-hop neighbor nodes, for each node and for each time slot, the cluster head can suggest a list of possible destination nodes. More specifically, consider two nodes $m$ and $n$ scheduled to transmit in time slot $k$. Also assume that nodes $m$ and $n$ are directly connected to a node $r$. Since neither $m, n$ or the cluster head knows whether $m$ and $n$ are NFNPs with respect to $r$, to be on the safe side the cluster head will advise nodes $m$ and $n$ not to transmit to node $r$. These nodes may choose to transmit to another node from the given list or to transmit a status packet to the cluster head.

\section{Discussion}

Note that our NF-TDMA algorithm is a combination of an optimal centralized solution with a distributed sub-optimal schedule. On the one hand, it uses available information at the cluster head in the form of network topology and NFNPs to optimally schedule status packets given the interference cancellation probability. On the other hand, our algorithm opportunistically allows nodes to transmit packets to their onehop neighbors while preserving the collision-free reception of status packets. In our NF-TDMA algorithm, we assume a global round-robin TDMA schedule where slots are determined based on the maximum propagation delay, which is in turn determined by the system transmission range. This schedule allows some tolerance to motion as the information exchanged between the nodes and the sink node is only in the form of one-hop neighbor lists. Naturally, additional information can be used to refine the schedule at the cost of sensitivity to motion and channel conditions. Since our NFTDMA only allocates transmission time slots, it can be readily implemented also on such an improved schedule.

Low communication overhead is one immediate benefit yielded by the combination of centralized schedule optimization and distributed transmission decisions. Specifically, our solution does not require nodes to share their time-varying transmission requirements, so that in turn the sink does not need to broadcast the packet type and destination for each scheduled transmission. This is because, in our solution, each node can build its own near-far list and does not need to explicitly share it with the other nodes. Instead, when forming the near-far matrix, the only overhead is represented by the transmission of one-hop connectivity lists by the nodes, a total of $(N-1) \cdot(N-1)$ bits, and by the transfer of the resulting optimized schedule, $\mathbf{S}$, from the sink to the nodes. To transmit $\mathbf{S}$, the sink is required to transmit a total of $(N-1) \cdot(N-1)$ probability values. In our implementation, each probability value is represented by 1 byte. This calculation does not include the overhead needed for obtaining the one-hop neighbor lists and the list of near-far node pairs, which is out of the scope of this paper and can be addressed with a per-node overhead of $\log (N)$ bits re-transmitted roughly $N$ times by methods such as, e.g., [35].

The fact that our algorithm serves both contention-free and opportunistic transmissions is utilized to increase throughput even in cases where the system does not have interference cancellation capabilities. In fact, in case a node already served the transmission of status packets, it can further utilize the channel to opportunistically transmit packets. This decision is 
performed distributedly, and the cluster head guides nodes to properly choose the destination of their opportunistic packets. As a result, although TDMA is traditionally convenient only when the packet transmission rate is high, our algorithm remains a convenient choice also when the packet transmission rate is low. The advantages of this hybrid feature of our algorithm come from a specific application of cross-layer network design: using available information of both the contentionfree and the opportunistic communication types, the overall performance can be improved. However, the distributed choice of the packet type has its drawbacks. Specifically, since the transmission of $i_{n}$ in slot $k$ is set randomly according to probability $S_{n, k}$, there is a chance that $\mathbb{P}\left[\mathcal{E}_{n, t, t_{m}}\right]$ in (12) is greater than the threshold $\Theta_{\mathrm{Tx}}$ while no transmission is issued. In this case, node $i_{n}$ will issue an opportunistic packet while constraint (2) is not fulfilled. However, this risk is low, as the exploitation of spatial reuse and of the near-far effect provides a large number of transmission slots to each node. In fact, we never encountered such occurrence in our extensive simulations, nor in the outcome of our sea experiment (see Section VI).

\section{NumeriCAL RESUlts}

In this section, we discuss the performance of our NFTDMA algorithm. We measure the performance in terms of throughput of status packets, $\rho_{\text {through,s }}$ from (1), scheduling delay of opportunistic packets, $\rho_{\text {delay,o }}$ from (4), and throughput fairness of opportunistic packets, $\rho_{\text {fair,o }}$ from (5). We show results for three configurations of the NF-TDMA protocol, namely: 1) Ideal NF-TDMA, where the interference cancellation probability is ideal and both the jammer and the jammed nodes of each NFNP are assumed to be decoded with probability 1 ; 2) Realistic NF-TDMA, where the interference cancellation probability is set according to the evaluated SINR; and 3) Limited NF-TDMA, where nodes do not have interference cancellation capabilities. ${ }^{6}$ The case of Limited NF-TDMA is further explored in our field experiment. For clarity, for all three schemes we do not apply the power control mechanism described in Section IV-B4. The performance of the power control refinement is explored instead in the results of the lake experiment in Section VI. We compare our results with the performance of the simple round-robin TDMA protocol (TDMA) where in each time slot only one node can transmit. For a fair comparison with the NF-TDMA algorithm, we duplicate the frame of the simple TDMA schedule to match that of the NF-TDMA schemes (i.e., $\left.L=T_{L} / T_{s}\right)$. Since we consider a star topology, where all nodes are directly connected to the cluster head node $i_{0}$, all other available spatial-reuse collision-avoidance protocols that use only topology information would converge to the simple TDMA protocol. This is because, without taking advantage of the near-far effect, a star topology means that the sink node would not be able to receive packets if simultaneous transmissions occur.

\footnotetext{
${ }^{6}$ Recall that, even with no interference cancellation capabilities, multiple nodes can transmit simultaneously if the destinations of their packets are different and certain interference conditions are satisfied.
}

\section{A. Simulation Setup}

Our simulation setup includes a Monte-Carlo set of 200 topologies. In each simulation run, $N=8$ nodes are placed uniformly at random in a volume of $5 \times 5 \mathrm{~km}^{2}$ with a water depth of $100 \mathrm{~m}$. The volume includes four horizontal obstacles and one vertical obstacle at uniformly distributed locations with uniformly distributed length in the range $[100,200] \mathrm{m}$. For each node pair in line-of-sight, we perform a Bellhop run [44, Ch. 3] for shallow waters of depth $100 \mathrm{~m}$, flat sand bottom, fixed sound speed $1500 \mathrm{~m} / \mathrm{s}$, and carrier frequency $10 \mathrm{kHz}$. The Bellhop model outputs a multipath structure from which the power attenuation level can be derived. Using the estimated power attenuation level, we calculate the SNR of the line-of-sight node pair considering a source level of $170 \mathrm{~dB}$ re $(1 \mu \mathrm{Pa}$ at $1 \mathrm{~m})$, a noise level of $40 \mathrm{~dB}$ re $\left(1 \mu \mathrm{Pa}^{2} / \mathrm{Hz}\right)$, and a transmission rate of $1000 \mathrm{bps}$. Then, for the calculated SNR level and considering BPSK communications at a transmission rate of 1000 bps, we set a link between a line-of-sight node pair if the bit error rate is less than $10^{-3}$. In case two or more packets are simultaneously received, the SNR level of each packet is used to calculate the signal-to-interference-plusnoise ratio (SINR) level of each packet, which in turn is used to calculate the bit error rate.

For status packets, we consider a target transmission rate of one packet every $T_{L}=100 \mathrm{~s}$, see (2). Considering available commercial acoustic modems [45], we set the maximum transmission range to be $3000 \mathrm{~m}$, corresponding to a maximum propagation delay of $2 \mathrm{~s}$. Then, considering the transmission of synchronization signals, a training sequence, 1000 data bearing bits, and guard time for possible clock drifts, we set the time slot to be $T_{s}=5 \mathrm{~s}$.

Each simulation run includes 1000 time slots. For each time slot $i$, a node $n$ for which $S_{n, i} \geq x$ where $0 \leq x \leq 1$ is drawn uniformly at random, can transmit either a status packet or an opportunistic packet. Status packets are always available and are transmitted to node $i_{0}$. The arrival times of opportunistic packets are distributed according to a Poisson process of rate $\lambda=1$ packet per minute per node, and these packets are stored in the node's local queue. The type of packet to be transmitted is determined via (13) in Section IV-B3, where we set $\Theta_{\mathrm{Tx}}=0.8$.

\section{B. Simulation Results}

In Fig. 3, we show the empirical complementary cumulative distribution function (C-CDF) of $\rho_{\text {through,s }}$ from (1) for status packets. Clearly, since TDMA does not depend on the specific network topology but only on the number of nodes, and since status packets are always available, the network throughput of TDMA changes negligibly across different runs. Compared to the performance of TDMA, we observe a significant improvement using our schemes, where even without interference cancellation capabilities (i.e., for Limited NFTDMA) the network throughput increases by $40 \%$, whereas with perfect interference cancellation the improvement can be as large as a factor of 4. Since no status packets collide at the cluster head when using Ideal NF-TDMA, the results are expectedly better than those for Realistic NF-TDMA. 


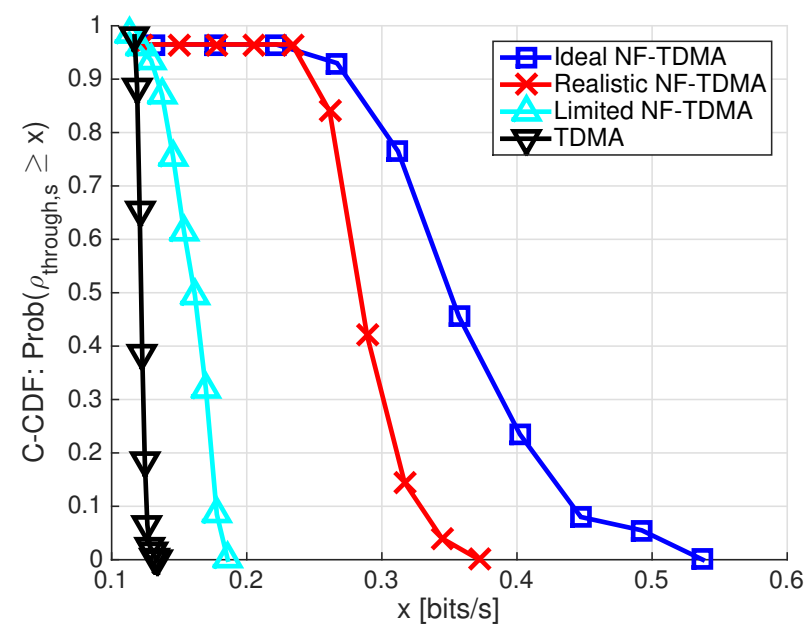

Fig. 3. Empirical C-CDF of $\rho_{\text {through,s }}$ from (1).

However, even for the latter, the network throughput improves by a factor of 3 compared to TDMA. We note that we are mainly concerned about the delay of opportunistic packets, as long as the minimum transmission rate of status packets is maintained, see (2). Hence, the opportunistic packets are scheduled whenever possible, and the resulting $\rho_{\text {through,s }}$ would not change considerably when using the refinement mechanism in Section IV-B4.

The empirical CDF results of $\rho_{\text {delay,o }}$ from (4) are shown in Fig. 4. Here we observe that, on average, the scheduling delay is roughly $30 \mathrm{~s}$ for TDMA. Since $L=T_{L} / T_{s}=20$ slots and $N=8$, this result comes directly from the fact that 1.5 opportunistic packets can be scheduled in each TDMA frame on average. We observe that interference cancellation capabilities improve the delay performance of Realistic NFTDMA and Ideal NF-TDMA by respectively 1.8 and 3.6 times, compared to TDMA. In addition, the results show that a more significant improvement exists for the Limited NF-TDMA scheme. This surprising result is because opportunistic packets are sent to one-hop neighbor nodes but not to the cluster head, and since the schedule only ensures collision-free reception of status packets, opportunistic packets are prone to collisions. Because in Limited NF-TDMA fewer opportunistic packets are transmitted than in Realistic NF-TDMA and Ideal NF-TDMA, fewer collisions occur, and the scheduling delay decreases.

To comment on the fairness in scheduling opportunistic packets, in Fig. 5 we show C-CDF results of $\rho_{\text {fair,o }}$ from (5). Since TDMA evenly allocates opportunistic packet transmissions, its fairness $\rho_{\text {fair,o }}$ is far better than that of the NFTDMA schemes. Not much difference is observed between the three different NF-TDMA schemes. Yet, Realistic NF-TDMA consistently outperforms Ideal NF-TDMA. This is because, as seen from the results of Fig. 3, the latter correctly allocates more opportunistic packet transmission opportunities to nodes located close to the cluster head. We also observe that the fairness of Limited NF-TDMA varies compared to that of Realistic NF-TDMA and Ideal NF-TDMA. This is because, in terms of fairness, the performance of Limited NF-TDMA strongly depends on the topology. Specifically, for a certain NFNP with respect to the cluster head, spatial reuse in Limited

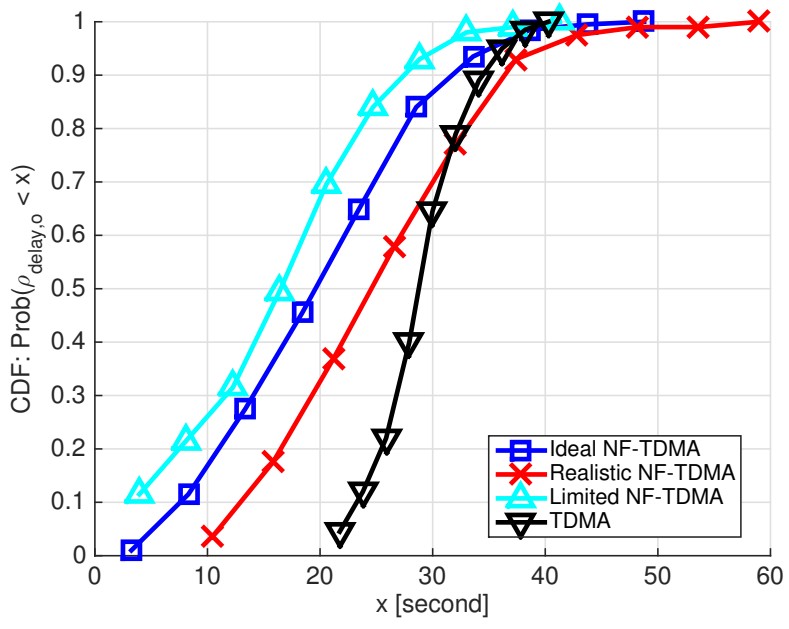

Fig. 4. Empirical CDF of $\rho_{\text {delay,o }}$ from (4).

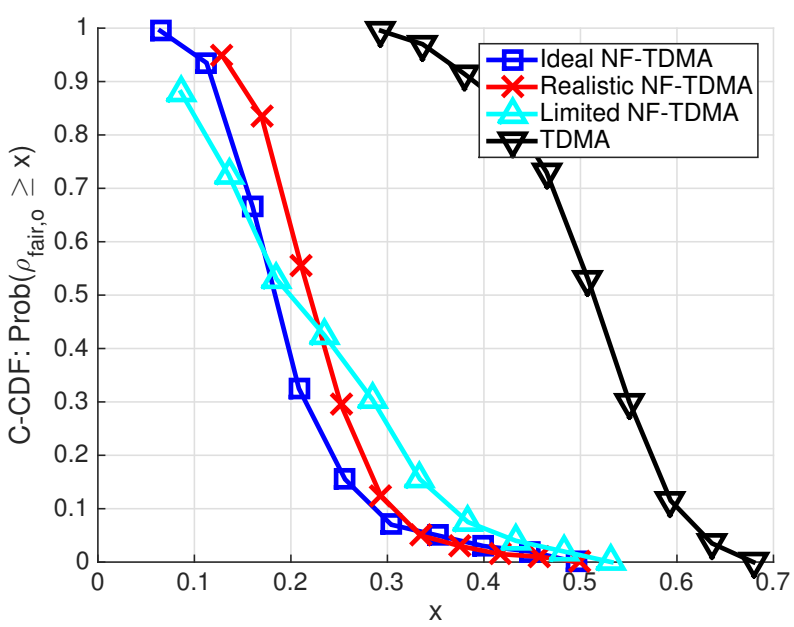

Fig. 5. Empirical C-CDF of $\rho_{\text {fair }, o}$ from (5).

NF-TDMA is determined by the ability of the far node to find a destination which is not connected to the near node. In some topologies, such destination nodes are found for only one or a few nodes, which adversely impacts fairness; conversely, in other topologies several far nodes can find proper destination nodes, and fairness improves as a consequence.

\section{FIELD EXPERIMENT}

In our numerical results, we showed the performance of the NF-TDMA algorithm in terms of network throughput, scheduling delay, and service fairness. To apply our simulations, we used a simple flat bathymetry. In addition, due to the use of this simple model and to avoid additional assumptions, in our simulations we did not consider the use of the refined scheme described in Section IV-B4. To verify our insights, in this section we present results from a field experiment using off-the-shelf acoustic modems with and without the power control mechanism. These modems do not have interference cancellation capabilities and can thus verify our conclusions for the interesting (and realistic) case of Limited NF-TDMA. The experiment also demonstrates the effectiveness and prac- 
ticality of our scheme, which can be easily implemented on top of any existing physical layer.

\section{A. Experiment Setup}

The setup of the experiment is shown in Fig. 6. The experiment was conducted in Dec. 2015, in lake Garda, Italy. The experiment included five nodes, communicating using EvoLogics modems. Our algorithm was implemented using the emulation capabilities of the ns2-based DESERT Underwater framework [46] and a real time synchronized scheduler connecting the application layer to the acoustic modems. The modems had no interference cancellation capability and in a near-far scenario can thus receive at most one packet. The nodes were deployed from harbor docks and from boats. By moving the two boats we created the four different network topologies shown in Fig. 6. Nodes 2 and 3 were deployed from harbor docks $75 \mathrm{~m}$ from each other and at water depth of $2 \mathrm{~m}$ and $4 \mathrm{~m}$, respectively (all topologies). Nodes 1 and 5 were placed $10 \mathrm{~m}$ from each other and were deployed from a small boat at water depth $10 \mathrm{~m}$ and distance $\sim 700 \mathrm{~m}$ from node 2 (Topologies 1, 2, 3) and at water depth $2 \mathrm{~m}$ and distance $\sim 50 \mathrm{~m}$ from node 2 (Topology 4 ). Node 4 was deployed from an additional boat at water depth $5 \mathrm{~m}$ and distance $200 \mathrm{~m}$ (Topology 1), $700 \mathrm{~m}$ (Topology 2), and $600 \mathrm{~m}$ (Topologies 3, 4) from node 2 . When the boats were distant from the harbor, the water depth was roughly $30 \mathrm{~m}$, where the water depth at the harbor was between 2 and $4 \mathrm{~m}$.

In each of the tested topologies, the network was fully connected. Node 1 was set as the designated cluster head. With respect to the cluster head, in Topology 1 node 5 was the jammer of NFNPs including nodes 2, 3 and 4. In Topology 2, both nodes 4 and 5 were the jammers of NFNPs including nodes 2 and 3, but $(4,5)$ was not a NFNP. In Topology 3, node 5 was jamming nodes 2,3 and 4 , and node 4 was jamming nodes 2 and 3. In Topology 4, nodes 2, 3 and 5 were jamming node 4 , and node 5 was jamming nodes 2 and 3. At the beginning of each topology test, to verify our setting, we performed a "discovery check" where we tested the links and the possible NFNPs. Then, the refined NF-TDMA algorithm (see Section IV-B4) was tested for 1000 s, followed by another $1000 \mathrm{~s}$ where we also tested the more conservative NF-TDMA algorithm in (10) (i.e., without power control). Nodes transmitting without power control used the maximum source level of $182.5 \mathrm{~dB}$ re $(1 \mu \mathrm{Pa} @ 1 \mathrm{~m})$, while nodes for which $S_{n, k}=n, n \neq 1$ transmitted using a source level of $162.5 \mathrm{~dB}$ re $(1 \mu \mathrm{Pa} @ 1 \mathrm{~m})$.

Taking into account the propagation delay and the delay of the modem for decoding the packets, we used a time slot duration of $T_{s}=5 \mathrm{~s}$. As a limitation to the rate of transmitting status packets, we considered $T_{L}=100 \mathrm{~s}$ (see (2)). The resulting TDMA frame contained $T_{L} / T_{s}=20$ time slots, and each run was configured to involve a total of 10 TDMA frames. In each of these frames, each node from 2 to 5 sent at least one status packet to node 1. As discussed in Sections IV-B3 and IV-B5, each node from 2 to 5 also sent several unicast opportunistic packets to other nodes in each TDMA frame. Both the status and the opportunistic packets included 64
TABLE I

SPECIFICATIONS OF THE EVOLOGICS S2RC 18/34 MODEM AND ENVIRONMENTAL INFORMATION.

\begin{tabular}{ll}
\hline Info & Value \\
\hline Carrier frequency & $26 \mathrm{kHz}$ \\
Bandwidth & $16 \mathrm{kHz}$ \\
Source level & 162.5 to $182.5 \mathrm{~dB}$ re $1 \mu \mathrm{Pa} @ 1 \mathrm{~m}$ \\
Processing delay & $0.15 \mathrm{~s}$ \\
Bit rate & $976 \mathrm{bps}$ \\
Estimated data rate & $512 \mathrm{bps}$ \\
Packet size & $512 \mathrm{bits}$ \\
Water temperature & 7 to $12{ }^{\circ} \mathrm{C}$ \\
Water salinity & $350 \mathrm{ppm}$ \\
Sound speed & $\approx 1448 \mathrm{~m} / \mathrm{s}$ \\
\hline
\end{tabular}

information bytes, which were transmitted at a rate of $500 \mathrm{bps}$, so that the total duration of each packet was roughly $1 \mathrm{~s}$. Due to the fully connected topology and the maximum range of $700 \mathrm{~m}$ between the nodes, this ensures that collisions are bound to occur whenever two nodes transmit in the same time slot.

Even though the lake surface was calm during the experiment, the very shallow water depth and the many reflections from the harbor's concrete walls resulted in a high overall packet error rate, which was measured to be roughly $20 \%$. As a result, the performance of the simple TDMA procedure ${ }^{7}$ is limited to the reception of 8 status packets by node 1 , and to a per-node reception of 32 opportunistic packets. The latter is calculated by taking into account that $(20-4) \cdot 3 / 4$ time slots are available at each node for reception of opportunistic packets in each TDMA frame, out of which on average $1 / 3$ are directed to the specific node and only $80 \%$ are successful. A summary of the relevant communication features and environmental characteristics experienced during the trial is reported in Table I.

\section{B. Experiment Results}

The results are shown in Table II. For each topology, we show the per-node number of status packets received (Column of Node 1), and the per-node number of opportunistic packets received (Columns of Node 2-5). For the opportunistic packets, in brackets we show the number of exposed terminal problems solved. These are the number of time slots a node was allowed to transmit beyond the guaranteed $c_{n}=2$ time slots (see (10)) in each TDMA schedule time frame. We note that the average per-node number of status packets received by node 1 was 8.5 and 6.5 with and without power control, respectively. We note that the numbers in Table II were obtained through offline processing of the reception logs of each node, and involved no additional overhead during network operations.

As expected, on average more opportunistic packets were received when power control was applied. This is supported also by the larger number of exposed terminal problems resolved when using power control. However, we observe that

\footnotetext{
${ }^{7}$ Recall that due to the fully connected network, simple TDMA is the method to which all spatial-reuse TDMA methods which do not utilize nearfar converge.
} 

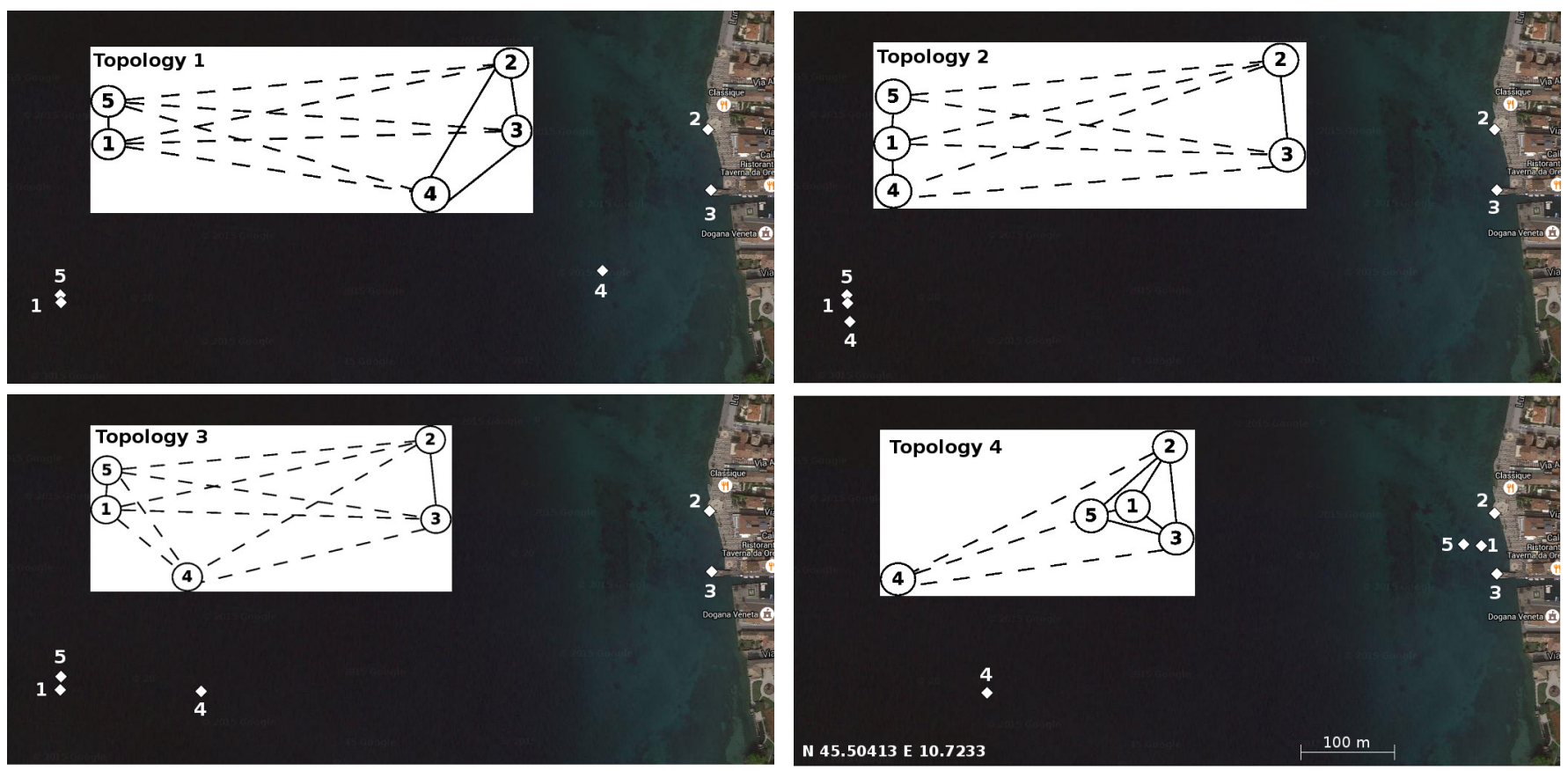

Fig. 6. Setting of the lake experiment. Geographical maps show the location of the nodes. The white panel in each map conveys the logical topology of the network. Near-far connections with respect to node 1 are shown using dashed lines. The bottom-left corner in all maps corresponds to the geographical coordinates $\left(45.50413^{\circ} \mathrm{N}, 10.7233^{\circ} \mathrm{E}\right)$. (Maps courtesy of Google Maps.)

in some cases better results are obtained without applying power control. This is because the modems supported a maximum source level reduction of $20 \mathrm{~dB}$, which was not always sufficient. As a result, interference occurred and packets were dropped. Compared to the expected results of TDMA, we note the significant increase in the average number of received opportunistic packets using the two versions of our NFTDMA algorithm. Namely, roughly $75 \%$ and $50 \%$ additional opportunistic packets were received with and without power control, respectively. Considering the results in Fig. 3 for limited interference cancellation, these experimental outcomes validate our simulations.

\section{CONCLUSIONS}

In this paper, we focused on the problem of transmission assignment in UWANs. We considered a time slot-based scheduling approach for a network topology where primary conflicts are not allowed and all nodes are directly connected to the sink. Exploiting the near-far effect, we proposed a spatial reuse scheduling solution that allows concurrent transmissions even when interference cancellation is not available. We formalized the problem of resource allocation for a given interference cancellation model and solved it optimally to achieve a collision-free scheduling solution while maintaining a minimum required packet transmission rate. Our scheduling algorithm is unique in the sense that it services both contention-free and opportunistic communications, each having different objectives. For the former, the objective is to maximize the network throughput, whereas for the latter scheduling delay and throughput fairness are of concern. Our numerical results show that in terms of all three objectives our schedule significantly outperforms the TDMA protocol, to which all current spatial-reuse scheduling protocols converge
TABLE II

LAKE EXPERIMENT RESULTS. NUMBERS INDICATE STATUS PACKETS RX BY NODE 1 AND OPPORTUNISTIC PACKETS RX BY ALL OTHER NODES. (IN PARENTHESES: NUMBER OF EXPOSED TERMINAL PROBLEMS SOLVED.)

\begin{tabular}{|c|c|c|c|c|c|c|}
\hline Topology & Method & Node 1 & Node 2 & Node 3 & Node 4 & Node 5 \\
\hline \multirow{2}{*}{ T1 } & $\begin{array}{l}\text { Power } \\
\text { control }\end{array}$ & 11 & $36(0)$ & $62(0)$ & $13(17)$ & $10(44)$ \\
\hline & $\begin{array}{l}\text { No Power } \\
\text { Control }\end{array}$ & 10 & $33(0)$ & $72(0)$ & $13(17)$ & $10(32)$ \\
\hline \multirow{2}{*}{$\mathbf{T 2}$} & $\begin{array}{l}\text { Power } \\
\text { control }\end{array}$ & 16 & $62(7)$ & 34 (19) & $44(49)$ & $43(52)$ \\
\hline & $\begin{array}{c}\text { No Power } \\
\text { Control }\end{array}$ & 9 & $35(7)$ & 59 (19) & $65(7)$ & $21(24)$ \\
\hline \multirow{2}{*}{ T3 } & $\begin{array}{l}\text { Power } \\
\text { control }\end{array}$ & 22 & $89(0)$ & $113(0)$ & $55(25)$ & $10(44)$ \\
\hline & $\begin{array}{c}\text { No Power } \\
\text { Control }\end{array}$ & 20 & $85(0)$ & $102(0)$ & $63(17)$ & $10(32)$ \\
\hline \multirow{2}{*}{ T4 } & $\begin{array}{l}\text { Power } \\
\text { control }\end{array}$ & 89 & $118(68)$ & $46(42)$ & $78(34)$ & $15(44)$ \\
\hline & $\begin{array}{c}\text { No Power } \\
\text { Control }\end{array}$ & 67 & $81(0)$ & $29(30)$ & $71(34)$ & $18(32)$ \\
\hline \multirow{2}{*}{ Avg } & $\begin{array}{l}\text { Power } \\
\text { control }\end{array}$ & \multicolumn{5}{|c|}{$56.5(27.8)$} \\
\hline & $\begin{array}{l}\text { No Power } \\
\text { Control }\end{array}$ & \multicolumn{5}{|c|}{47.9 (15.7) } \\
\hline
\end{tabular}

under the considered network topology. For the realistic case of no interference cancellation capability, we verified our results and demonstrated the effectiveness of our system in a field experiment. The results confirmed the high benefit of utilizing information about near-far node pairs to increase network throughput.

\section{REFERENCES}

[1] J. Heidemann, M. Stojanovic, and M. Zorzi, "Underwater sensor networks: applications, advances and challenges," Philosophical Transactions of the Royal Society of London A: Mathematical, Physical and Engineering Sciences, vol. 370, no. 1958, pp. 158-175, Jan. 2012. 
[2] T. Melodia, H. Kulhandjian, L. Kuo, and E. Demirors, "Advances in underwater acoustic networking," Mobile Ad Hoc Networking: Cutting Edge Directions, vol. 852, Mar. 2013.

[3] C. Fullmer and J.-J. Garcia-Luna-Aceves, "Solutions to hidden terminal problems in wireless networks," in Proc. ACM SIGCOMM, Cannes, France, Sep. 1997.

[4] M. Chitre, S. Shahabudeen, and M. Stojanovic, "Underwater acoustic communications and networking: Recent advances and future challenges," Marine Technology Society, vol. 42, no. 1, pp. 103-116, Apr. 2008.

[5] N. Chirdchoo, W.-S. Soh, and K. Chua, "RIPT: A receiver-initiated reservation-based protocol for underwater acoustic networks," IEEE $J$. Select. Areas Commun., vol. 26, no. 9, pp. 1744-1753, Dec. 2008.

[6] Y. Noh, U. Lee, S. Han, P. Wang, D. Torres, J. Kim, and M. Gerla, "DOTS: A propagation delay-aware opportunistic MAC protocol for mobile underwater networks," IEEE Trans. Mobile Comput., vol. 13, no. 4, pp. 766-782, Apr. 2014.

[7] R. Diamant, G. Shirazi, and L. Lampe, "Robust spatial reuse scheduling in underwater acoustic communication networks," IEEE J. Oceanic Eng., vol. 39, no. 1, pp. 32-46, Jan. 2014.

[8] D. Pompili, T. Melodia, and I. F. Akyildiz, "A CDMA-based medium access control for underwater acoustic sensor networks," IEEE Trans. Wireless Commun., vol. 8, no. 4, pp. 1899-1909, Apr. 2009.

[9] M. Zuba, Z. Shi, Z. Peng, J.-H. Cui, and S. Zhou, "Vulnerabilities of underwater acoustic networks to denial-of-service jamming attacks," Wiley Security and Communication Networks, vol. 8, no. 16, pp. 26352645, Nov. 2015.

[10] J. McGee, J. Catipovic, and P. Swaszek, "Leveraging spatial diversity to mitigate partial band interference in undersea networks through waveform reconstruction," in Proc. MTS/IEEE OCEANS, St.John's, NL, Canada, Sep. 2014

[11] P. Pandey, M. Hajimirsadeghi, and D. Pompili, "Region of feasibility of interference alignment in underwater sensor networks," IEEE J. Oceanic Eng., vol. 39, no. 1, pp. 189-202, Jan. 2014.

[12] R. Diamant, P. Casari, F. Campagnaro, and M. Zorzi, "A handshakebased protocol exploiting the near-far effect in underwater acoustic networks," IEEE Wireless Comm. Lett., 2016, online-first version.

[13] M. K. Park and V. Rodoplu, "UWAN-MAC: an energy-efficient MAC protocol for underwater acoustic wireless sensor networks," IEEE J. Oceanic Eng., vol. 32, pp. 710-720, Jul. 2007.

[14] Y. Zhong, J. Huang, and J. Han, "A TDMA MAC protocol for underwater acoustic sensor networks," in Proc. IEEE YC-ICT, Beijing, China, Sep. 2009.

[15] P. Casari, F. E. Lapiccirella, and M. Zorzi, "A detailed simulation study of the UWAN-MAC protocol for underwater acoustic networks," in Proc. MTS/IEEE Oceans, Vancouver, Canada, 2007.

[16] W. van Kleunen, N. Meratnia, and P. J. Havinga, "Simplified scheduling for underwater acoustic networks," Journal of Networks, vol. 8, no. 1, pp. 4-14, Jan. 2013.

[17] C. Yun, A.-R. Cho, S.-G. Kim, J.-W. Park, and Y.-K. Lim, "A hierarchical time division multiple access medium access control protocol for clustered underwater acoustic networks," Journal of Information and Communication Convergence Engineering, vol. 3, no. 3, pp. 153-166, Sep. 2013

[18] Z. Li, Z. Guo, H. Qu, F. Hong, P. Chen, and M. Yang, "UD-TDMA: A distributed TDMA protocol for underwater acoustic sensor network," in Proc. IEEE MASS, Macau, China, Oct. 2009.

[19] L. Hong, F. Hong, Z. Guo, and Z. Li, "ECS: Efficient communication scheduling for underwater sensor networks," MDPI Sensors, vol. 3, no. 3, pp. 2920-2938, Mar. 2011.

[20] P.-H. Huang, Y. Chen, B. Krishnamachari, and A. Kumar, "Link scheduling in a single broadcast domain underwater networks," in Proc. IEEE SUTC, Newport Beach, CA, Jun. 2010.

[21] D. Marinakis, K. Wu, N. Ye, and S. Whitesides, "Network optimization for lightweight stochastic scheduling in underwater sensor networks," IEEE Trans. Wireless Commun., vol. 11, no. 8, pp. 2786-2795, Aug. 2012.

[22] K. B. Kredo II and P. Mohapatra, "A hybrid medium access control protocol for underwater wireless networks," in Proc. ACM WUWNet, Montréal, Canada, Sep. 2007.

[23] L. Badia et al., "An optimization framework for joint sensor deployment, link scheduling and routing in underwater sensor networks," in Proc. of ACM WUWNet, Los Angeles, CA, Sep. 2006.

[24] K. Kredo, P. Djukic, and P. Mohapatra, "STUMP: Exploiting position diversity in the staggered TDMA underwater MAC protocol," in Proc. IEEE INFOCOM, Rio de Janeiro, Brazil, Apr. 2009.
[25] K. Kredo and P. Mohapatra, "Distributed scheduling and routing in underwater wireless networks," in Proc. IEEE GLOBECOM, Miami, FL, Dec. 2010.

[26] C.-C. Hsu, K.-F. Lai, C.-F. Chou, and K. Ching-Ju Lin, "ST-MAC: Spatial-temporal MAC scheduling for underwater sensor networks," in Proc. IEEE INFOCOM, Rio de Janeiro, Brazil, Apr. 2009.

[27] Y. Zhu, S. N. Le, Z. Peng, and J.-H. Cui, "DOS: Distributed ondemand scheduling for high performance MAC in underwater acoustic networks," UCONN CSE, Tech. Rep. UbiNet-TR13-07, Jul. 2013.

[28] J. Ma and W. Lou, "Interference-aware spatio-temporal link scheduling for long delay underwater sensor networks," in Proc. IEEE SECON, Salt Lake City, UT, Jun. 2011.

[29] L. Hong, F. Hong, B. Yang, and Z. Guo, "ROSS: Receiver oriented sleep scheduling for underwater sensor networks," in Proc. ACM WUWNet, Kaohsiung, Taiwan, Nov. 2013.

[30] Z. Wu, C. Tian, H. Jiang, and W. Liu, "Minimum-latency aggregation scheduling in underwater wireless sensor networks," in Proc. IEEE ICC, Kyoto, Japan, Jun. 2011.

[31] A. Shashaj, R. Petroccia, and C. Petrioli, "Energy efficient interferenceaware routing and scheduling in underwater sensor networks," in Proc. MTS/IEEE OCEANS, St. John's, Canada, Sep. 2014.

[32] M. Chitre, M. Motani, and S. Shahabudeen, "Throughput of networks with large propagation delays," IEEE J. Oceanic Eng., vol. 37, no. 4, pp. 645-658, Oct. 2012.

[33] P. Anjangi and M. Chitre, "Scheduling algorithm with transmission power control for random underwater acoustic networks," in Proc. MTS/IEEE OCEANS, Genova, Italy, May 2015.

[34] — , "Design and implementation of super-TDMA: A MAC protocol exploiting large propagation delays for underwater acoustic networks," in Proc. ACM WUWNet, Washington, DC, Oct. 2015.

[35] R. Diamant, R. Francescon, and M. Zorzi, "Efficient link discovery for underwater networks," in The Third Underwater Communications and Networking Conference (UComms), Lerici, Italy, 2016.

[36] R. Jain, W. Hawe, and D. Chiu, "A quantitative measure of fairness and discrimination for resource allocation in shared computer systems," Eastern Research Lab, Tech. Rep. DEC-TR-301, 1984.

[37] A. Othman, W. Abidin, H. Zen, K. Ping, and N. Zamhari, "Performance evaluation for network set up and nodes discovery protocol in underwater grid topology networks," in Proc. APSITT, Kuching, Malaysia, Jun. 2010.

[38] R. Diamant and L. Lampe, "Spatial reuse time-division multiple access for broadcast ad hoc underwater acoustic communication networks," IEEE J. Oceanic Eng., vol. 36, no. 2, pp. 172-185, Apr. 2011.

[39] R. Otnes, P. van Walree, H. Buen, and H. Song, "Underwater acoustic network simulation with lookup tables from physical-layer replay," IEEE J. Oceanic Eng., vol. 40, no. 4, pp. 822-840, Oct. 2015.

[40] R. Diamant, G. N. Shirazi, and L. Lampe, "Robust spatial reuse scheduling in underwater acoustic communication networks," IEEE $J$. Oceanic Eng., vol. 39, no. 1, pp. 32-46, Jan. 2014.

[41] B. Bollobas, Random Graphs, 2nd ed. Cambridge University Press, 2001, pp. 34-74.

[42] W. Zhang and R. E. Korf, "An average-case analysis of branch-andbound with applications: Summary of results," in Proceedings of the National Conference on AI (AAAI), 1992, pp. 545-550.

[43] D. R. Smith, "Random trees and the analysis of branch and bound procedures," Journal of the ACM (JACM), vol. 31, no. 1, pp. 163-188, Jan. 1984.

[44] F. Jensen, W. Kuperman, M. Porter, and H. Schmidt, Computational Ocean Acoustics, 2nd ed. New York: Springer-Verlag, 1984, 2nd printing 2000.

[45] P. Casari and M. Zorzi, "Protocol design issues in underwater acoustic networks," Elsevier Computer Communications, vol. 34, no. 17, pp. 2013-2025, Jun. 2011.

[46] P. Casari et al., "Open-source suites for the underwater networking community: WOSS and DESERT Underwater," IEEE Network, special issue on "Open Source for Networking: Development and Experimentation", vol. 28, no. 5, pp. 38-46, Sep. 2014. 